\title{
GEMEINSAMES SCHREIBEN IN DER FREMDSPRACHE: MUSTER, KREATIVITÄT UND DAS GLÜCK DES AUTORS ${ }^{1}$
}

\author{
KIRSTEN SCHINDLER \\ Universität Bielefeld
}

ABSTRACT. The following article aims at examining the question in how far the text patterns influences the text production process. In an experimental study, German and Polish Students of French were asked to write a letter of application for a stage at the French enterprise Danone in groups of two. The groups could be distinguished due to their state of knowledge concerning the conventions of a typical French letter of application. In the article, I will show the different states from their text product. Besides, I will then ask what this knowledge, or the lack of it, may mean for the text production process. At the end, the article will focus on a discussion concerning the question of teaching writing in L2 and the idea of providing foreign language student with text patterns for different communicative tasks.

\section{Ziel und Methode}

Wenn polnische oder deutsche Studierende der Romanistik einen französischen Bewerbungsbrief schreiben, dann entstehen systematisch verschiedene Produkte. Der entscheidende Unterschied besteht darin, dass sich die Texte der polnischen Studierenden an einem einheitlichen Textmuster orientieren, während die Texte deutscher Studierender dies nicht tun.

Das ist das erste auffallige Ergebnis einer Untersuchung zum Schreiben in der Fremdsprache, die im Frühjahr 1999 an den Universitäten Bielefeld und Poznań durchgeführt wurde. Paare von deutschen und polnischen Studierenden wurden im Rahmen eines Experimentes gebeten, auf eine (fiktive) Anzeige gemeinsam einen Bewerbungsbrief auf französisch zu schreiben. Ihre Interaktion wurde auf Video aufgezeichnet.

In meinem Beitrag möchte ich die Unterschiede zwischen den deutschen und polnischen Textprodukten herausarbeiten und diskutieren, wie die unter-

${ }^{1}$ Vielen Dank an Ulrich Krafft für das „Glück des Autors“. Ihm, Ulrich Dausendschön-Gay, Elisabeth Gülich, Teresa Tomaszkiewicz und besonders Katrin Lehnen sei an dieser Stelle für zahlreiche Anregungen gedankt. 
schiedlichen Realisierungen in der Interaktion entstehen. Es soll gefragt werden, welche Rolle Textmuster für die gemeinsame Produktion eines Textes spielen können und inwieweit sich diese Überlegungen für die Fremdsprachendidaktik nutzen lassen.

Textmuster sollen hier als typische, in der Sprachgemeinschaft akzeptierte Merkmale des zu erstellenden Textes als Vertreter einer bestimmten Textsorte verstanden werden (Dausendschön-Gay/Krafft 1996:259; Sandig 1997:26-27). Sie stellen in diesem Sinne konventionalisierte Lösungen wiederkehrender kommunikativer Aufgaben dar. Diese Lösungen unterliegen zeit- und interaktionsbedingten Veränderungen, z.B. bedingt durch die Einführung neuer Medien, können aber auch kulturspezifisch andere Ausprägungen zeigen (Pogner 1999).

Wie sich bei der Textproduktion der Rekurs auf Textmuster manifestiert, lässt sich anhand Daten zu Textproduktionsprozessen beschreiben. Dabei stellt die Methode des gemeinsamen Schreibens - hier in Form konversationeller Schreibinteraktionen - eine besonders geeignete Untersuchungsgrundlage dar ${ }^{2}$. Konversationelle Schreibinteraktionen sind Gespräche, die die Beteiligten zur Lösung einer Schreibaufgabe führen. Zwei oder mehr Personen haben die Aufgabe, gemeinsam einen Text zu produzieren. Sie sind an allen Phasen der Textproduktion gleichermaßen beteiligt und tragen für das gemeinsame Textproduktion gleichermaßen Verantwortung. Die Gespräche, die bei der Herstellung eines Textes entstehen, zeigen eine Vielzahl verschiedener Aushandlungsprozesse. SchreiberInnen müssen beispielsweise Formulierungsentwürfe ihren PartnerInnen gegenüber begründen. Sie müssen sich bei der Planung und Überarbeitung des Textes abstimmen. Neben der Textproduktion im engeren Sinne können diese Aushandlungen auch über die Verteilung und Zuständigkeit von Aufgaben, sowie die Abstimmung von Aufgabenaktivitäten und die Effektivität von Kooperationstätigkeiten geführt werden. Dass sich aus diesen Aushandlungen Rückschlüsse über die Orientierung an Textmusterwissen ableiten lassen, soll die Analyse zeigen.

Im folgenden wird das Untersuchungsdesign vorgestellt und einzelne Versuchsparameter wie TeilnehmerInnen und Aufgabenstellung beschrieben. Anschließend wird vorgestellt, wie sich die skizzierten Unterschiede in den Textprodukten manifestieren können. Daran anknüpfend wird anhand ausgewählter Transkriptausschnitte diskutiert, wie sich der Einfluss von Textmusterwissen bei der Orientierung der Dyaden auswirken kann. Der Beitrag schließt mit der Frage, inwieweit die Kenntnis von Mustern zur Zufriedenheit von AutorInnen beitragen kann.

${ }^{2}$ Mit konversationellen Schreibinteraktionen wurden eine Reihe von Aspekten der Textproduktion systematisch bearbeitet. Siehe u.a.: Dausendschön-Gay/Gülich/Krafft 1992; Dausendschön-Gay/Krafft 1996; Bouchard/de Gaulmyn 1997; Dausendschon-Gay/Krafft 1999; Lehnen 1999; Lehnen 2000. 


\section{Untersuchungsdesign}

Paare von miteinander bekannten Französisch Studierenden des gleichen Studienjahres wurden aufgefordert, auf der Grundlage einer fiktiven Unternehmensanzeige des französischen Unternehmens Danone, einen Bewerbungsbrief zu schreiben und sich damit für ein zwei- bis sechsmonatiges Praktikum zu bewerben. Den Studierenden war nicht bekannt, dass es sich um eine fiktive Annonce und damit auch um ein fiktives Praktikumsangebot handelte. Die den VersuchsteilnehmerInnen zugrundegelegte Anzeige von Danone entsprach sowohl äußerlich, als auch inhaltlich dem Unternehmensimage, wie es sich im Internet präsentiert. Sie enthielt eine Reihe von Informationen zur Unternehmensgeschichte, der jetzigen Struktur des Unternehmens und seiner Produktpalette. Um den Entscheidungsspielraum der Studierenden nicht zu weit einzuschränken, wurden aber weder Angaben zum Inhalt des Praktikums, noch zu einer Vergütung, oder zum genauen Qualifikationsprofil der BewerberInnen gemacht. ${ }^{3}$ Stattdessen sollte durch eine ungenaue Angabe des Umfangs (zweibis sechsmonatiges Praktikum) und des Arbeitsortes (Paris oder Lyon) zusätzliches Entscheidungspotential und damit gleichzeitig Begründungszwang für die BewerberInnen entstehen. Danone wurde gewählt, weil es ein großes, weltweit operierendes französisches Unternehmen darstellt und davon auszugehen ist, dass seine Produkte sowohl den polnischen als auch den deutschen Studierenden bekannt sind. Die Aufgabe der Studierenden bestand konkret darin - auf der Grundlage der Anzeige - gemeinsam einen Bewerbungsbrief auf französisch zu formulieren, worin sie angeben sollten ,ce que vous attendez de votre stage et où vous expliquez pourquoi vous êtes le parfait complément pour notre team jeune et motivéc (Zitat aus der Anzeige). Die Kommunikationssprache innerhalb der Interaktion wurde freigestellt. Für die Aufgabe hatten die Studierenden 45 Minuten Zeit, wobei diese Zeitvorgabe nie unterschritten und im Einzelfall bis zu $1 \frac{1}{2}$ Stunden überschritten wurde. Zur Lösung der Aufgabe konnten sie auf ein- und zweisprachige Lexika zurückgreifen.

An die erste Aufgabe schloss sich unmittelbar eine zweite Schreibaufgabe an. Die Studierenden sollten sich um eine finanzielle Unterstützung ihres Praktikums bei dem an ihrer jeweiligen Universität verorteten Leonardo da Vinci Programm ${ }^{4}$ bewerben. Der Brief sollte in ihrer Muttersprache - also auf deutsch oder polnisch - abgefasst werden und ca. eine Seite lang sein. Für diese Auf-

${ }^{3}$ Als Voraussetzungen wurde die Sprachkompetenz: parlant couramment le français, der Berufsstand: jeune étudiant européen und das persönliche Profil der BewerberInnen: entreprenante, ouvert et curieux genannt (siehe Anzeige im Anhang).

${ }^{4}$ Das Leonardo da Vinci Programm ist ein Praktikantenprogramm der EU, das sich auf die Zusammenarbeit zwischen Hochschulen und Wirtschaft spezialisiert hat. Es vergibt Stipendien für Praktika und hilft bei der Organisation. 
gabe standen 30 Minuten zur Verfügung. Auch diese Zeitvorgabe wurden in den meisten Fällen überschritten, die Bearbeitungszeit blieb dennoch deutlich kürzer als bei der ersten Aufgabe. Beide Schreibinteraktionen wurde mit einer Kamera und zum Teil mit externem Mikrofon aufgezeichnet.

Im Anschluss an die Aufgaben wurde ein kurzes gemeinsames Gespräch mit der Versuchsleiterin geführt, in dem das Gelingen der Aufgabe in Hinblick auf die Textprodukte und die gemeinsame Schreibinteraktion diskutiert wurde. Anschließend wurde den Studierenden ein vierseitiger Fragebogen ausgehändigt, der Sozialdaten, Fremdsprachen- und Textsortenkenntnisse sowie Bewerbungserfahrungen abfragte, und den sie entweder sofort oder zu Hause ausfüllen konnten. Insgesamt nahmen an dem Experiment 22 Schreibpaare teil, wobei 18 Paare die beschriebene Aufgabe lösten. Die fünf deutschen Paare der Universität Bielefeld waren aus dem fünften bis zwölften Semester und zwischen 22 und 31 Jahre alt. Die 20 polnischen TeilnehmerInnen, die an der Universität Adama Mickiewicza in Poznań studierten, befanden sich zwischen dem zweiten bis fünften Studienjahr und waren zwischen 21 und $26 \mathrm{Jahre}$ alt $^{5}$. Daneben nahmen drei „gemischte“ Gruppen an dem Experiment teil: ein deutsch-polnisches, ein deutsch-französisches und ein polnisch-französisches Schreibpaar.

\section{Versuchsparameter: TeilnehmerInnen und Aufgabe}

Schreiben in der Fremdsprache wurde bisher in unterschiedlichen Zusammenhängen und anhand verschiedener Fragestellungen untersucht. Kontrastive Analysen der Textprodukte von SchreiberInnen, die Texte in ihrer Erst- bzw. Zweitsprache produzieren, waren insbesondere mit dem Ziel verknüpft, die Schreibfertigkeiten der FremdsprachenlernerInnen zu verbessern. Eingebunden in den universitären Kontext, wurden diese Untersuchungen vor allen Dingen für das wissenschaftliche Schreiben durchgeführt. Das in der Anfangszeit vorherrschende Dogma, aus den fremdsprachlichen Textprodukten auf Denkstile abzuleiten und diese zu bewerten (vgl. Kaplan 1966), ist in der Folgezeit stark relativiert worden (Clyne 1987; Purves 1988; Connor 1992; Clyne 1993). Die spezifischen Bedingungen und Prozesse des Schreibens in der Fremdsprache sind insbesondere mit introspektiven Methoden, wie dem Lauten Denken, erforscht worden (Krings 1986; Krings 1989). Ein wichtiges Ergebnis dieser Untersuchungen ist, dass SchreiberInnen bei der Textproduktion in der Fremdsprache zwar spezifischen Problemen ausgesetzt sind, diese sich aber unabhängig

${ }^{5}$ Die größere Anzahl von polnischen Paaren ist durch einen längeren Forschungsaufenthalt an der Adam-Mickiewicz-Universităt in Poznań begründet. An dieser Stelle möchte ich dem DAAD für die Unterstützung während meines Auslandsaufenthaltes im Rahmen seines DoktorandInnenprogrammes danken. 
von ihrer Schreibkompetenz stellen, die sie auch in ihrer Erstsprache besitzen. Gemeinsames Schreiben in der Fremdsprache von Angehörigen unterschiedlicher Erstsprachen stand im Mittelpunkt einer Reihe von Untersuchungen konversationeller Schreibinteraktionen (Dausendschön-Gay/Krafft 1996; Dausendschön-Gay/Krafft 1999). Neben der Untersuchung spezifischer Aspekte der Interaktion, stehen hier insbesondere Formulierungsverfahren im Mittelpunkt der Analyse. Inzwischen sind auch Arbeiten zum domänenspezifischen Schreiben in der Fremdsprache erschienen, die insbesondere den Fokus auf kulturspezifische Aspekte der Textproduktion richten (Büchle 1992; Tiittula 1995; Adamzik/Antos/Jakobs 1997; Pogner 1999).

In meiner Untersuchung sollen kulturspezifische Aspekte weitgehend ausgeblendet werden und durch eine Beschreibung zweier Gruppen von FremdsprachenlernerInnen ersetzt werden. Diese Gruppen unterscheiden sich in ihrer Erstsprache (Polnisch bzw. Deutsch) verfügen aber über gleiche Kenntnisse in einer gemeinsamen Zweitsprache (Französisch). Damit sollten für die Gruppen gleiche Bedingungen etabliert werden. Diese Bedingungen stellten sich in der Untersuchung allerdings als deutlich unterschiedlich dar. Bedingt durch einen sehr viel umfangreicheren Sprachpraxisanteil in ihrem Studium, besitzen die polnischen Studierenden ein Wissen über verschiedene Textmuster zur Lösung unterschiedlicher kommunikativer Aufgaben. Sie sind daher im Rahmen ihrer Ausbildung für die Aufgabe, ein Bewerbungsschreiben auf französisch zu schreiben, vorbereitet worden. Anders dagegen die deutschen Studierenden, die zwar auch in der Fremdsprache schreiben müssen, aber dies vorwiegend für die wissenschaftliche Textproduktion einüben (rédaction avancée) ${ }^{6}$.

Eine weitere Bedingung des Experimentes bestand darin, innerhalb der Schreibpaare gleiche Voraussetzungen zu schaffen. D.h. beide SchreiberInnen sollten die gleiche Erst- und die gleiche Zweitsprache besitzen, das gleiche Fach (Romanistik) studieren und sich im gleichen Studienabschnitt befinden. Damit sollte eine bereits vor Beginn des Experimentes etablierte Zuständigkeit für bestimmte Aufgaben vermieden werden. Die Einschränkung auf professionelle

${ }^{6}$ Der Anteil an sprachpraktischen Pflichtveranstaltungen „Apprentissage de la langue française" ist in der Studienordnung der Universität Adama Mickiewicza (UAM) mit durchschnittlich 7 SWS vom ersten bis zum fünften Studienjahr deutlich höher veranschlagt als in der Universität Bielefeld (durchschnittlich 2 SWS, allerdings bereits inklusive Phonetik und Syntax Kursen, die in der UAM als eigene Kurse angeboten werden und noch zusätzlich 6 SWS umfassen). Außerdem sind anders als an der Universität Bielefeld in der UAM alle für Romanistik angebotenen Veranstaltungen auf französisch, die Studienarbeiten müssen auf französisch angefertigt werden. Siehe die Studienordnung der Universität Bielefeld: www.lili.uni-bieleeld.de/ seiler/ordnungen/romanistik.html und www.lili-uni-bielefeld.de/ seiler/ordnunen.franzoesisch.html. Zur Studienordnung der Universität Adama Mickiewicza siehe: Institut de philologie romane. Catalogue d'informations des spécialités: philologie romane, philologie espagnole, philologie italienne, philologie roumaine. Poznań, année universitaire 1999/2000. 
FremdsprachenlernerInnen war von der Hypothese geleitet, dass sie einen reflektierten und damit kritischen Umgang mit eigenen fremdsprachlichen Textprodukten gewöhnt sind, und sich daher beim Schreiben stärker mit Normen und Konventionen von Textsorten auseinandersetzen, als es bei anderen Gruppen von SprachlernerInnen zu erwarten ist.

Die Textsorte „Bewerbung" wurde aus verschiedenen Gründen ausgewählt. Zunächst lässt sich ein Bewerbungsschreiben in relativ kurzer Zeit produzieren und bedarf in einer Experimentsituation keiner weiteren Vorbereitungen, Recherchen oder Hilfsmittel. Daneben ist der Bewerbungsbrief eine Textsorte, die im Alltag der Studierenden vorkommt. Als übliche kommunikative Aufgabe sich für eine Tätigkeit zu bewerben, verfügen die Studierenden entweder bereits uber Erfahrungen mit Bewerbungsschreiben oder werden in naher Zukunft diese Erfahrungen machen. Zuletzt ist der Bewerbungsbrief eine Textsorte, die in einer reichhaltigen Auswahl von Ratgeberliteratur ausführlich behandelt wird. In den unterschiedlichen Ratgebern verschiedener Länder (Frankreich, Deutschland, Polen) werden anhand von Musterbriefen und Negativbeispielen bestimmte musterhafte Realisierungen vorgestellt. Als Mindestvoraussetzungen einer Bewerbung sollen hier gelten: eine Realisierung und mögliche Spezifizierung des Sprechaktes sich bewerben und die deutliche Einbettung des Sprechaktes in einen Absender-Empfänger Zusammenhang.

Ein Bewerbungsschreiben ist aber auch noch in anderer Hinsicht interessant. Eingebunden in eine asymmetrische, formelle und fernkommunikative Schreibsituation (vgl. Drescher 1994:120) erfordert der Bewerbungsbrief auf der einen Seite ein genaues Wissen um sprachlich adäquate Formen und Muster, verlangt aber andererseits eine individuelle Lösung im Hinblick auf die Darstellung der Motivation und eigenen Person. So ist es für den Bewerber notwendig, in einem ersten direkten Kontakt mit dem potentiellen neuen Arbeitgeber nicht nur zu zeigen, dass er über eine entsprechende Qualifikation für die ausgeschriebene Position verfügt, sondern auch, dass er konventionalisierte Formen der schriftlichen Briefkommunikation beherrscht. Damit lässt sich der Bewerbungsbrief als vorgeformter Text charakterisieren, dem ein klares Textmuster unterliegt. ${ }^{7}$ Weil die BewerberInnen sich aber in einer Konkurrenzsituation befmden, muss sich der Brief gegenüber anderen durch eine individuelle und möglicherweise kreative Lösung absetzen. In dem Spannungsfeld zwischen Formelhaftigkeit und Kreativität verortet, variiert der jeweilige Grad von erlaubter Kreativität bzw. erwünschter Formelhaftigkeit des Bewerbungsbriefes dabei je nach Berufssparte, aber auch nach kulturspezifischen und individuellen Vorlieben (Hempelmann 1997:88-89). Wenn eine Bewerbung in der Fremd-

${ }^{7}$ Zur Diskussion um Vorgeformtheit und Formelhaftigkeit siehe: Gülich 1997 und Gülich/Krafft 1998. 
sprache abgefasst werden muss, wird es zusätzlich schwierig, den ,richtigen Ton" zu treffen. Nicht nur sprachliche Probleme, beispielsweise durch die Unkenntnis vorgeformter Ausdrücke in der Fremdsprache, auch kulturspezifische Unterschiede wie beispielweise Fragen der Höflichkeit (vgl. Brown/Levinson 1987) können die - hier gemeinsame - Formulierungsarbeit erschweren ${ }^{8}$.

\section{Textmusterorientierung}

\subsection{Die Textprodukte}

In einem ersten Schritt möchte ich nun die Textprodukte der Studierenden einem systematischen Vergleich unterziehen. Dabei interessiert mich besonders, ob den Texten unterschiedliche Textmuster zugrunde liegen, wie sich die Textmuster in den Texten manifestieren und in welcher Hinsicht sich diese Muster unterscheiden.

Insgesamt sollen für die Analyse 15 Bewerbungsbriefe untersucht werden, die in entsprechenden Konstellationen (polnisch-polnische bzw. deutsch-deutsche Interaktionen) entstanden sind. Ein erster Untersuchungsaspekt bildet die äußere Form und Struktur der Briefe. In einem zweiten Schritt sollen dann spezifische Formulierungsvorschläge und inhaltliche Aspekte, auf die damit verwiesen wird, im Fokus der Analyse stehen.

Form und Struktur der Briefe: Ein erster Unterschied zwischen den polnischen und deutschen Bewerbungsbriefen ${ }^{9}$ manifestiert sich in der Anzahl der verschiedenen Versionen, die in der Interaktion produziert werden. Die polnischen Studierenden stellen in der Regel zwei und bis zu fünf Versionen ihrer Bewerbung her, wobei die letzte Version eine handschriftlich saubere Abschrift der letzten Version darstellt, in der alle Spuren der gemeinsamen Formulierungsarbeit getilgt sind. Die deutschen Studierenden produzieren hingegen in drei von fünf Fällen nur eine Version. In zwei Fällen waren zusätzlich Konzeptpapiere erstellt worden. In allen deutschen Endversionen sind aber noch deutlich Korrekturen und Revisionen durchgeführt worden. Geht man nicht davon aus, dass nur die polnischen Studierenden dem Gebot der Höflichkeit Folge leisten, indem sie einen lesbaren und sauberen Text für die Versuchsleiterin produzieren, so muss diese unterschiedliche Einstellung in Hinblick auf das zu

${ }^{8}$ Einen systematischen Vergleich von Bewerbungsschreiben in verschiedenen europäischen Ländern und den in Deutschland üblichen Formen hat Limbrunner (1999) durchgeführt.

${ }^{9}$ Der Einfachheit halber soll hier von polnischen und deutschen Textprodukten gesprochen werden. Wobei die polnischen Texte die Texte polnischer Studierender, die deutschen Texte die deutscher Studierenden sind. 
erzeugende Produkt andere Schlussfolgerungen zulassen. Erstens: den deutschen Studierenden ist es nicht wichtig, das Textprodukt formalen Ansprüchen gemäß zu „stylen“. Gemäß dem deutschen Muster, wie es in Ratgebern vorgestellt wird, wird der Bewerbungsbrief üblicherweise auf dem Computer erstellt. $\mathrm{Da}$ in der Experimentsituation diese Möglichkeit nicht vorgesehen war, konnten die deutschen Studierenden die Bewerbung nicht entsprechend „fertig“ machen $^{10}$. Zweitens: Im polnischen Muster ist eine handschriftliche Bewerbung üblich, weshalb die Frage der Fertigstellung innerhalb der polnischen Dyaden keine Probleme aufwirft, die polnischen Texte haben bereits in ihrer handschriftlichen Form ihren Bearbeitungsendpunkt erreicht. Damit verweist das polnische Muster auf die Bewerbungsrealität in Polen und gleichzeitig auf die traditionelle französische Bewerbung, die handschriftlich üblich war und zum Teil immer noch ist (Hempelmann 1997:19-20) ${ }^{11}$.

Allen polnischen Texten ist gemeinsam, dass die Absenderadresse links oben plaziert ist, wobei die Empfängeradresse rechts auf gleicher Höhe und zum Teil etwas niedriger zu finden ist. Allen Texten polnischer Studierender ist auch gemeinsam, dass anschließend eine Anrede folgt. Das eigentliche Textkorpus, das sich wiederum bei allen Produkten gleicht, lässt sich durch deutliche, eingerückte Paragraphen beschreiben und wird durch eine Verabschiedungsformel und die Unterschrift abgeschlossen. Die polnischen Studierenden haben also eine Vorstellung über formale Konventionen der französischen Bewerbung und sie haben eine einheitliche Vorstellung davon.

Solche eindeutigen, formalen Übereinstimmungen lassen sich in den deutschen Texten nicht wiederfmden. In zwei Fällen lässt sich das beschriebene Adressenmodell wiederfinden, in zwei Fällen ist die Empfängeradresse links unter der Absenderadresse verortet. In einem Fall fehlen beide Adressen. Strukturierende Paragraphen sind in zwei Fällen, in drei Fällen nicht ersichtlich. Hier ist es auf einer formalen Ebene nicht möglich von einem einheitlichen Muster zu sprechen, das den deutschen Briefen zugrunde liegt. Das wird auch durch weitere Ergänzungen des Textkorpus deutlich, die sich in den polnischen Briefen so nicht wiederfmden lassen. Die deutschen Studierenden geben in drei Fällen einen Betreff- bzw. Bezug an, in zwei Fällen wird darauf verzichtet. Aber auch da, wo sich die Briefe deutscher Studierender vermeintlich gleichen - bei der Betreffzeile, lassen sich Unterschiede feststellen. Im ersten Fall haben

${ }^{10}$ In den deutschen Interaktionen wird das Problem, dass keine Abschrift auf dem Computer möglich ist, mehrfach angesprochen.

${ }^{11}$ Die polnische Bewerbungsliteratur zeigt sich hier deutlich weniger einheitlich als beispielsweise die deutsche. Bis 1990 gab es in Polen ăhnlich wie in allen sozialistischen Ländern keine Bewerbung entsprechend dem skizzierten Muster. Die polnische Bewerbungsliteratur übernahm daher die in England, Frankreich und Deutschland tradierten Textmuster übersetzte sie in der Regel ins Polnische (vgl. Gajewska 1998:274-280; Kienzler 1999). 
sich die beiden Studentinnen auf Candidature pour un stage geeinigt. Eingeleitet mit Sujet: (die korrekte Form wäre in diesem Fall object gewesen) funktioniert die Betreffzeile als Überschrift oder Titel des Briefes.

\section{Sujet: Candidature pour un stage}

Madame, monsieur,

je vous écris en réponse à votre annonce dans le magazine régionale "Hallo Halle! ". J'aimerais poser ma candidature pour un stage chez votre entreprise.

Deutlich wird dies auch, wenn der erste Fall mit einem zweiten Beispiel kontrastiert wird. Im zweiten Beispiel fehlt das eigentliche Nennen des Betreffs (object) und der Ausdruck ist durch die Zeitangabe erweitert: Candidature pour un stage de six mois. Anders als im ersten Fall ersetzt hier der Betreff den Sprechakt Bewerbung, der anschließend nicht mehr realisiert wird.

\section{Candidature pour un stage de six mois}

Mesdames et Messieurs,

je suis une jeune étudiante du français de 29 ans et je m'intéresse à votre offre d'un stage. Je terminerai mes études en juin, c'est à dire qu'à partir de juillet je serai libre à compléter votre team.

Im dritten Fall wird sowohl das eben beschriebene Vorgehen wiederholt (der Betreff ersetzt den Sprechakt sich bewerben) als auch durch die Erweiterung des Betreffs mit einem Bezug eine Kommunikationssituation zwischen Bewerberin und Unternehmen hergestellt.

candidature pour un stage d'étudiant

votre annonce dans le monde du 20 mars 1999

Mesdames/Messieurs, je suis intéressée à faire un stage dans l'entreprise Danone.

An diesen Ausschnitten wird deutlich, dass ähnliche Formulierungen von den Studierenden unterschiedlich genutzt werden. Andersherum verhält es sich im nächsten Fall. In zwei der drei deutschen Briefe, die auch schon eine Betreffzeile formuliert haben, wird am Ende des Briefes auf Anlagen verwiesen.
Annexe
- curriculum vitae
Pièces jointes - curriculum vitae
Certificats
- certificat du Bureau Müller à Bonn

Zwar werden mit annexe und pièces jointes unterschiedliche sprachliche Formen aufgegriffen, sie besitzen aber die gleiche Funktion und werden in der französischen Bewerbungsliteratur auch synonym benutzt, wobei pièces jointes als traditionelle und annexe als moderne Formulierung beschrieben wird. Die inhaltliche Einheitlichkeit wird hier durch unterschiedliche sprachliche Formen 
realisiert. Auffällig ist, dass die Gruppen, die bereits einen Betreff des Briefes realisiert hatten, auch auf Anlagen verweisen und damit eine erweiterte Briefform wählen. Beide Gruppen geben im Fragebogen an, dass sie bereits über Erfahrungen mit deutschen Bewerbungsschreiben verfügen, aber nur wenig bzw. gar keine Erfahrungen mit französischen Bewerbungsschreiben haben. Damit scheinen die Studierenden in Deutschland übliche Musterbestandteile (Betreffzeile, Anlagenverweis) auf den französischen Brief zu übertragen.

Inhaltliche Aspekte und ihre sprachlichen Formen: Bei einer Analyse inhaltlicher Aspekte der Briefe fallen trotz individueller Variationen zwei gravierende Unterschiede auf. Erstens: die Briefe polnischer Studierender verfügen ohne Ausnahme über Informationen, die die direkte Charakterisierung der eigenen Person betreffen. In den deutschen Briefen fehlen diese Angaben bis auf eine Ausnahme. Zweitens: in neun der zehn polnischen Briefe wird ein direkter Bezug zum Unternehmen und seinen Produkten hergestellt, in lediglich zwei der fünf deutschen Briefe wird ebenso vorgegangen, wobei die sprachlichen Formen deutlich zurückhaltender sind, als bei den Texten polnischer Studierender:

So wird in zwei Briefen deutsch-deutscher Paare formuliert:

1) Un stage chez vous, comme entreprise alimentaire de premier plan, serait une occassion excellente pour améliorer mes compétences dans ces deux domaines.

2) J'étais très impressionnée de l'importance de votre entreprise dans l'industrie alimentaire mondiale.

Die polnischen Paare versuchen den Bezug zum Unternehmen so darzustellen:

1) Je voudrais avouer que j'ai toujours rêvé de travailler dans une entreprise qui respecte ses consommateurs, augmente la qualité de ses produits et de son service.

2) ... je trouve votre proposition de stage très intéressante, à plus forte raison que l'image de votre entreprise a depuis longtemps attiré mon attention.

3) Personellement, je suis enthouisiaste de vos produits qui sont de très haute qualité sur le marché polonais. Danone est aujourd'hui connu dans le monde entier et reconnu comme une entreprise numéro un parmi les autres sociétés laitières.

4) J'ai choisi le Groupe Danone parce qu'il est un leader de l'industrie alimentaire mondiale.

5) J'apprécie beaucoup ses produits qui sont présent dans tous les pays du monde. Pour ses nombreuses qualités je suis curieuse et ouverte de connaître votre entreprise extraordinaire.

An diesen Beispielen lässt sich zweierlei zeigen. Zunächst greifen alle Schreibpaare auf Informationen der Anzeige zurück. Während die deutschen Studieren- 
den mit ihren Angaben aber Unternehmenskenntnis ausdrücken und dies gleichzeitig als Chance für ihre berufliche Zukunft ausweisen, gehen die polnischen Studierenden einen Schritt weiter. Ihre Strategie kann als ,flatterie ${ }^{\text {cc }}$ bezeichnet werden. Indem sie auf die Bedeutung des Unternehmens und seiner Produkte in Bezug auf ihre Person eingehen und auf ihr sich bereits lange abzeichnendes Interesse verweisen, geben sie diesen Informationen einen persönlichen Anklang und eine fast vertraute Note - ein Tabu in den Briefen deutscher Studierender. Wie in den Besprechungen deutlich wurde, wird das uneingeschränkte Loben von Produkten und/oder Unternehmen von den deutschen Studierenden als unpassend abgelehnt. Dies deckt sich auch mit Hinweisen in deutschen Ratgebern, die den BewerberInnen in dieser Hinsicht deutliche Vorsicht raten. Sowohl in den Texten polnischer Studierender als auch in den Textmustern der französischen Ratgeberliteratur wird dieser Aspekt weniger kritisch gehandhabt.

Noch deutlicher manifestieren sich Unterschiede in den Briefen deutscher und polnischer Studierender, wenn es um Fragen der Selbstcharakterisierung bzw. des Selbstlobs geht. Unter Selbstcharakterisierung soll der Aspekt der Selbstdarstellung verstanden werden, der Informationen über persönliche Eigenschaften z.T. mit Hilfe wertender Adjektive enthält und der mit den biographischen Angaben und beruflichen Erfahrungen zusammen das Bild eines Kandidaten ausmacht (Hempelmann 1997, Anhang, i-ii). Auffällig ist das bereits konstatierte fast durchgängige Fehlen von selbstcharakterisierenden Passagen in den deutschen Briefen. Einzig in einem deutschen Brief beschreibt die Kandidatin sich selbst als jeune, motivée, je n'ai pas de problèmes $m$ 'adapter à des nouvelles circonstances et de $m$ 'exposer à des provocations. Etant étudiante, je suis habituée à travailler en team, mais aussi à résoudre des problèmes indépendamment. Ähnlich wie das Unternehmenslob ist auch die Selbstcharakterisierung bei den deutschen Studierenden tabuisiert und nur sehr vorsichtig gebraucht. Dies wird auch daran deutlich, dass - obwohl in der Anzeige direkt auf charakterliche Qualifikationen abgezielt wurde - auf diese nicht eingegangen wird. In der Uneinheitlichkeit der deutschen Briefe lässt sich also doch so etwas wie eine Regelmäßigkeit erkennen, die sich durch Normen wie die Vermeidung von direktem Unternehmens- oder Selbstlob charakterisieren lässt und damit auf die deutsche Bewerbungsliteratur verweist. Anders in den polnischen Briefen. Auch wenn mit dem Selbstlob möglicherweise ebenso kritisch umgegangen wird, ist dieser Aspekt konstituierender, musterhafter Bestandteil des Schreibens, wobei sich auffällige Regelmäßigkeiten zeigen.

Je suis une personne dynamique et entreprenante

Je suis une personne ouverte qui entre facilement en contact... Entreprenante, énergique je suis prête à accepter ...

Jeune, dynamique et pleine d'enthousiasme...

Ma spontanéité et mon optimisme $m$ 'aident à entrer facilement en contact avec 
les gens. Toujours pleine de nouvelles idées, énergique et disponible... Je m'estime une personne ouverte...

Ensuite, je suis une personne qui prend facilement le contact avec des gens... Je suis une personne ouverte et entreprenante

... je sais coopérer avec les autres, je suis dynamique, active

J'entre facilement en contact avec les gens...

Comme je suis ouverte et $j$ 'ai le goût du contact humain ... mon esprit créatif

Alle Beteiligten stellen heraus, dass sie über die in der Anzeige geforderten Bedingungen verfügen. Dabei übernehmen sie für ihre Formulierungen zum großen Teil die in der Anzeige benutzen Adjektive oder entsprechende Synonyme. An den wiederkehrenden Formulierungen lässt sich zeigen, dass die Selbstcharakterisierung akzeptierter Teil des polnischen Briefmusters ist, dass wiederkehrende Formulierungen auf gelerntes Wissen der Studierenden in ihrem Fremdsprachenunterricht verweisen und dass drittens die in der Anzeige geforderten Bedingungen Autorität besitzen.

Wie weit hier polnische und deutsche Vorstellungen auseinanderliegen, zeigt sich in der Interaktion zwischen einer deutschen und einer polnischen Studentin: Roberta (deutsche Studentin aus Bielefeld) und Paula (polnische Studentin aus Poznań) haben sich darauf geeinigt, dass Paula sich bewirbt. Sie hat schon ihre persönlichen Angaben und ihre Studiumsentwicklung skizziert und ist nun dabei sich zu überlegen, was noch in den Brief hineingehört. ${ }^{12}$

P: maintenant pourquoi je je m'intéresse à $C A^{\prime}$... ăh.. qu'est-ce que $\mathrm{j}^{\text {‘'attends }}$

$\mathrm{R}$ : mmh est-ce que $\mathrm{t}^{6}$ es déjà assez ((betont)) entreprenante

$\mathrm{X}$ : $P$ zählt mit ihren Fingern, eins, zwei R zeigt mit dem kleinen Finger auf die Anzeige

P: oui il faut un peu écrire ((stöhnt)) (7) bah oui c'est SÛR qu'il faut écrire quelquechose pas vraiment

R: ouvert et curieux' +

$\mathrm{X}$ : Blick zu P, P guckt in die Luft, denkt nach

P: ouvert et curieux faire ... sinon il faut un peu trichER dans cette lettre

$\mathrm{X}$ : P grinst, $R$ lacht, gucken sich an

6 Minuten später stellt sich wieder die gleiche Frage, nachdem ausführlich etwas zu Erfahrungen und Sprachqualifikation geschrieben wurde.

P: okay mainteNANT' äh que jsuis ((laut)) la MEILLeure + candidate pour le boulot

$\mathrm{R}$ : où bien tu veux

$\mathrm{X}$ : P grinst, guckt zu R, R zur Tür

${ }^{12}$ Die Transkriptionssymbole finden sich im Anhang. Es wurde die französische und deutsche Schriftsprache benutzt. 
Roberta versucht ihr anschließend zu erklären, dass es an dieser Stelle wichtiger ist darauf einzugehen, warum sie das Praktikum machen will. Der nächste Ausschnitt ist ca. 5 Minuten später

P: je suis.. äh ((schnell)) entreprenante,..+ pourquoi' ((leise))

R: ((schnell)) ah NON ça tu veux pas éCRIRE LÁ parce que c'est dans l'anNONCE

$\mathrm{X}$ : Ps Blick geht zur Anzeige und schreibt, $R$ hat das beobachtet und guckt jetzt auch zur Anzeige, dann zu $P$

P: non jsais pas' ((leise)) tu penses, non on ne peut pas'+

$R$ : c'est dans l'anNONCE, non il faut le monTRER' par des... parce que ce que tu as

$\mathrm{X}$ : P lehnt sich zurück, verzieht den Mund

P: ah d'accord, $\mathrm{j}$ 'ai jamais écrit une lettre de candidature

R: FAIT moi non PLUS ((gedehnt)) MAIS + si c'est là äh

$\mathrm{X}$ : P lächelt, dann $R, R$ zeigt auf die Anzeige

R: normalement $c^{\prime}$ est pas dans ... ((leise)) je ne sais pas + mais si c'est dans l'aNNONce comme $\mathrm{CA}^{\prime}$ ' je crois pas si.. on

$\mathrm{X}$ : guckt zu P, P zur Anzeige

P: oui d'accord... moi j'écrirerais mais.. que jsuis

R: peut reCOpier tout simplement bah vaS-Y' $Y^{c} . c^{c}$ est ta lettre à toi

$\mathrm{X}$ : $P$ streicht entreprenante durch

P: ouverte et la meilleure ((leise)) donc pas meilleure + mais ce qui j'attends de ce stage ah..

R: tu pourrais peut-être formu

$\mathrm{X}$ : $P$ zeigt mit dem Stift auf die Anzeige; $R$ guckt zu $P$, dann zur Tür

$\mathrm{R}$ : le autreMENT. tu pourrais dire que tu aimes bien assumer des responsabiliTES ăh que tu aimes connaître des

$\mathrm{X}: P$ guckt zu $R$ und abwechselnd zur Anzeige

P: mmh, mmh okay..

$\mathrm{R}$ : nouveaux GENS et que tu aimes travailler avec d'autres mais seulement pas ăh le mot MÊME

Roberta weist zunächst ironisch darauf hin, dass Paula sich noch nicht als entreprenante, ouvert et curieux dargestellt hat. Paula reagiert nicht auf die Ironie der Äußerung. Vielmehr behandelt sie die Selbstdarstellung als ernstzunehmende kommunikative Aufgabe. Dies hängt damit zusammen, dass es für sie Teil des Musters ist. Nachdem Roberta zunächst den Versuch Paulas, sich als geeignete Kandidatin herauszustellen, abgewehrt hat, kommt Paula einige Minuten später wieder darauf zurück. Sie will für die Aufgabe, sich zu charakterisieren, auf die in der Anzeige benutzte Formulierung (vous êtes entreprenante) zurückgreifen. Dies ruft einen starken Protest Robertas hervor. Ihr erster Hinweis, man solle sich nicht direkt darstellen, sondern dies indirekt zeigen, wird im Laufe der Interaktion darauf beschränkt, dass Paula nicht das in der Anzeige 
benutzte Adjektiv verwenden soll. Beide TeilnehmerInnen leisten hier deutliche Beziehungsarbeit, um den Konflikt unterschiedlicher Auffassungen abzumildern. Deutlich wird aber auch, dass Roberta nicht genau sagen kann, weshalb die in der Anzeige benutzte Formulierung nicht übernommen werden soll (je ne sais pas mais si c'est dans l'annonce comme ça je ne crois pas). Sie ist sich dennoch sicher, dass dieses Vorgehen nicht günstig ist und Paula dafür die Verant-

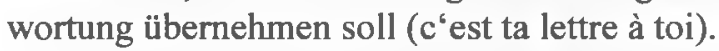

Die polnischen Briefe folgen also einem Muster, das formale, inhaltliche und sprachliche Aspekte umfasst. Die deutschen Briefe, die sich von den polnischen in verschiedener Hinsicht unterscheiden, lassen kein einheitliches Muster erkennen, wenn auch Übereinstimmungen z.B. in der Ablehnung von Selbstlob existieren. Außerdem zeigt sich bei den deutschen Briefen eine Orientierung am deutschen Bewerbungsmuster. Wie auch in dem Abschluss eines deutschen Briefes zu erkennen ist:

Pour des informations complémentaires, je serai toujours à votre disposition. En attendant votre réponse positive je reste avec mes salutations sincères. [verbleibe ich mit freundlichen Grüßen] ${ }^{13}$

\subsection{Ausschnitte aus den Interaktionen}

Was bedeutet die Kenntnis von Mustern für das gemeinsame Schreiben? Welche Auswirkungen hat die Musterorientierung für die Aushandlungsprozesse in der Interaktion, für Formulierungsvorschlãge und Kooperationstätigkeiten? Diese Fragen sollen an verschiedenen Schreibanfänge untersucht werden, wo die Beteiligten gemeinsame Schreibgrundlagen herstellen.

Beispiel 1. Ein Muster abrufen: Dijana und Alexandra, zwei polnische Studentinnen aus dem vierten Studienjahr, haben die Anzeige gelesen. Alexandra fängt an mit Hilfe Dijanas, die Briefformalia zu produzieren. Nach der Erledigung dieser Aufgabe nimmt sich Dijana Stift und Papier. Alexandra schließt ihren Stift.

A: ((leise)) alors... parlant couramment le français, entreprenante, ouvert et ăha, äha

D: un jeune étudiant parlant couramment le français, entreprenante, ouvert et curieux de connaître cette

$\mathrm{X}$ : D liest die Anzeige laut vor, A steigt ein

A: mmh à lettre

${ }^{13}$ Besonders auffallig ist an dieser Stelle der kurze Absatz, der im französischen Briefmuster nicht vorgesehen ist. 
D: entreprise extraordinaire, .. de parfait complément pour notre team jeune et motivé, āha.. donc .. à la réponse de votre

X: jeweiligen Stellen in der Anzeige; D schaut nicht mehr aufs Blatt,

A: suite de vo ((betont)) suite de votre annonce

D: suite de ((betont)) suite de votre annonce

$\mathrm{X}$ : sondern zu $A, A$ schaut auch zu $D$; beide lächeln, A schaut wieder auf ihr Blatt und öfnet ihren Stift

Anschließend an die Realisierung der Briefformalia, stellt sich den polnischen Studentinnen die Aufgabe, den Bezug herzustellen. In auffälliger Weise wenden sich die Partnerinnen in dem Moment einander zu, sie scheinen sich zu signalisieren, dass eine neue Aufgabe ansteht. Beide haben für den Bezug (formule $d^{\prime}$ attaque) eine Anzahl von Formulierungen gelernt und sind nun gemeinsam dabei die hier passende zu formulieren. Dass sie die hier passende Formulierung gefunden haben, bestätigen sich die beiden mit einem Lächeln und dem Öffnen des Stiftes. Sie signalisieren einander die Bereitschaft, den Satz aufschreiben zu wollen.

Beispiel 2. Wenn das Muster nicht funktioniert: Martina und Anja, zwei polnische Studierende aus dem dritten Studienjahr, haben beide die Anzeige gelesen. Anja hat vorgeschlagen, dass sie Martina helfen möchte, Martina sich also bewerben soll. Die Anzeige liegt für beide ersichtlich in der Mitte. Martina hat sich fürs Aufschreiben gerüstet.

A: en se referant à votre annonce parue

Nach diesem ersten Vorschlag, der so sofort von beiden akzeptiert wird und von Martina aufgeschrieben wird, schließt sich eine längere Debatte darüber an, woher die Anzeige stammen könnte. Nachdem beide konstatiert haben, dass es keine Hinweise darüber gibt wird die Formulierungsarbeit wieder aufgenommen. In dem längeren Transkriptausschnitt wird deutlich, wie sehr fehlende Angaben Irritationen in der Dyade auslösen können.

$\mathrm{M}$ : donc. en se referant à votre annonce parUE donc ${ }^{6}$ (4) parue donc,...tt mmh

$\mathrm{X}$ : A schlägt vor, einfach drei Punkte zu machen. Blick zu A

$\mathrm{M}$ : concernant,.. à la candidature pour un stage à votre annonce parue donc,.. concernant. ANNONCE

A: à en se referant à votre annonce parue $\mathrm{mmm}$ je

A schüttelt den Kopf, zeigt auf Ms Blatt, liest laut, beide gucken

M: en se

A: voudrais poser ma candidature,.. ((leise)) pour un stage, comme ça $+(7)$ ((leise)) se referant à votre annonce

$\mathrm{X}$ : abwechselnd auf Ms Blatt und sich an 
M: referant à votre annONce, parue donc $\mathrm{mmm}$. concernant la candidature pour un stage chez danON.. je pose,..

$\mathrm{M}$ : en se referant à votre candidature où je voudrais ((leise)) telle et telle je voudrais poser ma cand ma candi

A: je voudrais poser ma candidature candidature

$\mathrm{X}$ : einen nicht verständlichen Einwurf $M$ beginnt mit Selbstdiktat

$\mathrm{M}$ : candidature pour un stage... pour un stage.. pour un stage. chez danone,

A: à un pour un stage à ((betont)) pourvoir + atha

A grinst uber ihre Formulierung

Der Briefanfang: en se referant à votre annonce bildet in diesem Fall eine gemeinsame Grundlage der weiteren Formulierungsarbeit. Diese wird aber erheblich dadurch gestört, dass nicht so wie die Studentinnen es gelernt haben, eine Angabe über die Zeitung/Zeitschrift gemacht wird, an die dann der folgende Satz anknüpfen könnte. Auch hier lassen sich wieder deutliche gemeinsame Formulierungsgrundlagen erkennen, die in der Produktion ganzer Satzfragmente liegen und einen Hinweis darauf geben, dass vorgeformte Strukturen, an denen der Brief sich orientieren kann, bekannt und gelernt sind, auch wenn sich hier eine falsche Variante etabliert hat (me référant). Die Irritationen der Beteiligten in Bezug auf eine fehlende Angabe über den Erscheinungsort der Bewerbung zeigen, dass die gemeinsame Arbeit an einem Muster orientiert ist, bei dem entsprechende Angaben eine Voraussetzung darstellen, die nicht fehlen darf (Erscheinungsort). Die erwünschte Einhaltung des Musters wird dadurch erschwert, dass die Angaben fehlen.

Beispiel 3. Was man in dem Praktikum überhaupt machen kann? Ein ganz anderes Vorgehen lässt sich beim nächsten Beispiel beobachten. Anna und Clara, zwei Studentinnen aus Bielefeld kurz vor Abschluss ihres Studiums, haben beide die Anzeige und die Versuchsanleitung gelesen. Clara schiebt die näher bei ihr liegende Versuchsanleitung in die Mitte, so dass sie jetzt oberhalb der Anzeige liegt und für beide Teilnehmerinnen beide Texte gut sichtbar plaziert sind. Der Transkriptausschnitt zeigt ein für die deutschen Dyaden typisches Problem der anfänglichen Orientierungslosigkeit hier u.a. durch fehlende Angaben in der Anzeige begründet.

A: ((leise)) oKAY...+ also nen lebenslauf brauchen wir nich' also höchstens in einem text dann

C: ((leise)) mmh.. okay..ja+ nee du sollst

A atmet laut aus gucken sich an, $C$ guckt auf die Anzeige, A dann auch

A: ganz kurz zum beispiel, mmh

C: ja nur son brief schreiben,.. lettre de motivation.. ja.. und ähm.. man soll ja wahrscheinlich dann auch auf die

C: AnNONce eingehen. Also.. mmh (6) also eine idEE wär ja zum beispiel dass 
man sagt äh dass man das eben alles

$\mathrm{X}$ : Versuchsanleitung, A guckt in die Luft, C markiert eine Stelle auf der Annonce, A guckt jetzt auch auf die Annonce

A: und LOben natülich das is ja GANZ toLL

$\mathrm{C}$ : NICH gewusst hat ăh was sie genAU (8) ((leise)) ja+ also ich würd sagen das DU

$\mathrm{X}$ : A lacht, dann gemeinsam + lachen beide lesen

A: wegen STUwi ${ }^{14}$ und weswegen' was hast du gesacht"

$\mathrm{C}$ : das eher machst äh wegen Ly wegen stuwi, wegen lyON zum beispiel das

$\mathrm{X}$ : $C$ und $A$ gucken sich an, $A$ lächelt, dann auch $C$

$\mathrm{C}$ zeigt auf die Anzeige

A: mmh aber ich hab ja noch nich ganz

C: du da natürlich die GRÖßere motivation hast würd ich sagen..((leise)) okay,..+

$\mathrm{X}$ : lachen Blick von $\mathrm{A}$ zur Anzeige, dann zu C

A: verstanden was man, jetzt in dem praktikum machen KANN also außer das jetzt genauer kennen lernen (7) was das

$\mathrm{X}$ : beide gucken konzentriert auf die Anzeige

A: jetzt genau IS für nen praktikum... mmh

C: ich denke mal da is ja ăhm relativ LAnge und das man da durch alle MÖglichen

A: (5) ja is halt nur schwierig zu begründen. warum man jetzt dieses

$\mathrm{C}$ : abteilungen geht, .also wenn man nen halbes jahr da is"

A zeigt auf die Anzeige

A: praktikum machen will wenn man nich ne genaue vorstellung hat was das SEN soll ((schnell)) ich kann ja schlecht

A: schreiben ja das is so SCHÖN in LYon ich möchte gern wieder hin aber es reicht wohl schon allein dass

C: nee ähm (7)

X: lachen, $A$ guckt zu C, $C$ auf die Anzeige, $A$ jetzt auch

A: man dass ich stuwi mache vielleicht ${ }^{6}$ dass ich das deswegen dass ich da halt mein praktikum mache

$\mathrm{C}$ : ja, ja, und das du beides $\mathrm{X}$ : Blickkontakt zwischen $\mathrm{C}$ und $\mathrm{A}$

A: $\mathrm{mmh}$

C: vereinen willst also einerseits wirtschaftinter, in einem wirtschaftsunternehmen arbeiten und dann französisch

A nimmt sich einen Stift und beginnt mit dem Schreiben

Auffällig ist, dass der Aushandlungsprozess - wer sich bewerben soll - hier sehr deutlich an inhaltlichen Fragen orientiert ist, etwas, was sich in den polnischen Videoaufnahmen kaum wiederfinden lässt. Hier wird von Clara begründet, dass Anna sich bewerben soll, weil sie ,besser passt“. Ein Vorschlag, den

${ }^{14}$ Stuwi ist die Abkürzung für das an der Universität Bielefeld angebotene Zusatzstudienprogramm „Studierende und Wirtschaft", das für Studierende im Hauptstudium angeboten wird und ihnen Wirtschaftskenntnisse vermitteln soll. 
Anna auch sofort akzeptiert. Damit wird der Brief aber gleichzeitig auf sie abgestimmt. Bevor Anna mit dem Schreiben anfängt, will sie für sich grundsätzliche Fragen klären. Sie weiß nicht genau, wie ein Bewerbungsbrief aussieht, weiß aber, dass zumindest kein tabellarischer Lebenslauf erwünscht wird. Die mangelnde Kenntnis eines Musters spiegelt sich auch im weiteren Verlauf der Interaktion wider. Beide Teilnehmerinnen geben auch im Fragebogen an, dass sie weder über Erfahrungen mit deutschen noch mit französischen Bewerbungsschreiben verfügen. Annas grundlegendes Problem ist hier, dass sie nicht versteht, was sie in diesem Praktikum machen kann. Dieses Wissen benötigt sie aber, um die an sie gestellte Aufgabe zu erfüllen. Auch Claras erster Vorschlag, im Praktikum verschiedene Abteilungen kennen zu lernen, ist ihr keine Hilfe. Nachdem sie für sich geklärt hat, dass sie das Praktikum mit ihren eigenen Interessen begründen kann, ist sie bereit, mit dem Aufschreiben zu beginnen. Es erfolgen hier keine Aushandlungen, wer das Aufschreiben übernehmen soll, Anna übernimmt wie selbstverständlich die Schreibaufgabe. Dies ist umso erstaunlicher, weil in den polnischen Videos keine solche Selbstverständlichkeit $\mathrm{zu}$ beobachten ist. Schreib- und Bewerbungsrolle sind in den polnischen Dyaden in der Mehrzahl nicht an eine Person gebunden. Das Aufschreiben erfolgt meist abwechselnd nach erstellten Versionen oder persönlichen Möglichkeiten (schreibt schöner). Zum Teil sind aber auch keine Erklärungen oder RegelmäBigkeiten zu finden, so wird gemeinsam an einem Text oder gleichzeitig an verschiedenen Texten gearbeitet. Die Rolle des Aufschreibens bildet bei den polnischen Dyaden ebenso Aushandlungsbedarf wie die Frage nach der Rolle der BewerberIn. Anders als bei den deutschen Dyaden, wo in allen Fällen mit der Entscheidung, wer sich bewirbt, auch die Entscheidung darüber getroffen wird, wer den Text aufschreiben soll.

Beispiel 4. Den Kontext herstellen: Karoline und Cora zwei deutsche Studentinnen im Hauptstudium haben die Anzeige gelesen und sich darauf geeinigt, französisch zu sprechen, um die Briefproduktion in der Fremdsprache zu erleichtern. Die Anzeige liegt in der Mitte. Cora hat zu Beginn zwei Verständlichkeitsfragen (brouillon und raturer) gestellt, die ihr Karoline beantwortet hat. Auch hier zeigen sich ähnliche Orientierungsprobleme wie in dem letzten Ausschnitt.

Ka: ah. ce que je comprends pas dans cette annonce là'. c'est ce qu'on. ce qui attend les gens qui ont fait le stage,. parce

$\mathrm{X}$ : beide gucken hauptsächlich auf die Anzeige, nur jeweils kurze Blicke zueinander

Ka: que c'est un stage de QUOI' qu'est qu'on fait' (4) dans la fabrication,. dans les bureaux,. ou je jsais pas où est-ce

$\mathrm{X}$ : Kas Blick zu C und auf die Anzeige, $C$ guckt währenddessen auf die Anzeige 
Ka: que tu vois'. un stage comme

C: on ne dit rien.. mais je pense äh. ici on parle de six mois donc äh. on ne peut pas faire

$\mathrm{X}$ : C zeigt auf die Anzeige

C: grande chose .. äh . peut-être äh. mais.. faire partie d'un d'un petit projet mais.. si. äh ça dure plus äh plus longue

$\mathrm{X}$ : Cs Blick zu Ka, Blickkontakt wird nur kurz aufrechterhalten immer wieder Blicke zwischen $C$ und $K a$

Ka: ouiais

C: longue c'est un peu difficile ((schnell)) mais peut-être ähm ... on doit décider qui ath qui veut appliquer'

$\mathrm{X}$ : und wieder Konzentration auf die Anzeige

$\mathrm{Ka}$ : ((schnell)) donc c'est toi qui veut être étudiant qui pose sa candidature parce que je ne sais pas trop ce que je

C: d'accord

$\mathrm{X}$ : Ka schließt sofort an

Ka: veux faire chez danone ((schnell)) si tu veux... ((betont)) bien-sûr .donc ce que je

C: aha d'accord ((leise) et toi tu M'AIdes' ((leise)) d'accord

Ka: me demande si c'est à nous de PREciser ce qu'on veut faire là, si ((schnell)) $c^{\prime}$ 'est tellement OUvert + la. l'annonce

$\mathrm{Ka}$ zeigt auf die Anzeige; $\mathrm{Ka}$

Ka: ((schnell)) donc c'est à toi de de voir ce que tu FAIS de te PREsenter de dire jsuis. telle et telle personne et je fais

$\mathrm{X}$ : guckt zu C, C guckt die ganze Zeit auf die Anzeige

$\mathrm{Ka}$ : ça et ça et $\mathrm{j}$ 'aimerais bien faire ça chez vous + ,. donc je crois ça serait mieux quand tu dises ((betont)) pas du tout +

$\mathrm{X}$ : kurzer Blick von Ka zur Anzeige, wieder zu C, C ist weiterhin auf die

$\mathrm{Ka}$ : äh,.. où ils veulent te poser

$\mathrm{X}$ : Anzeige konzentriert; nach einer längeren Pause (8 Sek.) schlägt C vor, ein Konzeptpapier zu erstellen

Karoline schlägt vor, selbst den Kontext zu schaffen und genau zu beschreiben, was in dem Praktikum getan werden soll. Eine Aufgabe, die mit über zwei Stunden zu einem erheblichen Zeitaufwand führt. Karoline weist Cora die Aufgabe zu, sich zu bewerben und begründet dies mit ihrem mangelnden Interesse. Cora erwartet daraufhin von Karoline, die Aufgabe der Helfenden zu übernehmen. Damit sind auch die Zuständigkeiten innerhalb der Interaktion, ähnlich wie in dem obigen Beispiel, des Aufschreibens und der Verantwortlichkeit (c'est à toi de voir, quand tu dises) klar, die bei Cora liegen. Das erinnert auch an das erste Beispiel, wo Roberta darauf aufmerksam macht, dass es Paulas Brief ist und sie deshalb die Entscheidungsgewalt, aber auch die Verantwortung hat (c'est ta lettre à toi). Was hier aber auch auffällt, ist, dass Karoline eine Vorstellung darüber hat, was in den Brief gehört (présenter, faire chez vous). Cora 
bestätigt ihr dieses Muster aber nicht sofort, wie in den polnischen Beispielen gesehen. Die stärker monologischen Passagen machen deutlich, dass hier zunächst einsames Wissen über die Textsorte, den Kontext u.a. ausgetauscht werden muss. Der Wissenstand ist bei den deutschen Studierenden nicht wie bei den polnischen Studierenden gleich und im Rahmen der Hochschulausbildung gewonnen. Sie haben kein gemeinsames Wissen über die Textsorte Bewerbung und können in ihrer stärker individuell zusammengesetzten Ausbildung auch unterschiedliche fremdsprachliche Schreib-, aber auch Bewerbungserfahrungen gesammelt haben. Durch diese ungleichen Schreibvoraussetzungen stellt sich ihnen also eine deutlich andere Aufgabe als den polnischen Paaren, deren Arbeit über ihr gemeinsames Musterwissen koordiniert wird. Karoline und Cora wissen noch nicht, wohin die gemeinsame Arbeit geht, und wie sie zu einer Lösung gelangen. Sie müssen dafür stärker als die polnischen Paare ihre Vorstellungen austauschen. Für Karoline und Cora erhält die Anzeige als Grundlage eines um sie herum zu erstellenden Kontextes eine Schlüsselstellung und wird zum gemeinsamen Bezugspunkt der Arbeit.

Beispiel 5. Eins, zwei, drei - ein Bewerbungsbrief entsteht: In einem letzten Beispiel sollen die bereits beschriebenen Beobachtungen ergänzt werden. Katharina und Magdalene, zwei Studentinnen aus Poznań im vierten Studienjahr, haben die Anzeige gelesen und sich darauf geeinigt, dass sich Magdalene bewerben, Katharina das Aufschreiben übernehmen soll. Die Briefformalia werden gemeinsam relativ schnell produziert, wobei das erste Problem auftaucht, an wen man den Brief richten soll. Nachdem in der Anzeige keine Person direkt genannt ist, stimmen sie in Anlehnung an die im Sprachenunterricht bei ihrem französischen Lektor gelernte Form überein, Monsieur le directeur zu schreiben. Dieses Beispiel zeigt, wie sehr von gemeinsamen und damit austauschbaren Wissen gesprochen werden kann: Magdalena beginnt mit ihren Vorschlag, Katharina beendet und ergänzt ihn.

M: ((leise)) alors. donc.. ${ }^{+}$en fait on ne met pas je m'APPELLE telle lalanon mais je crois que

Ka: ça dépend comment tu veux écrire ça parce que

$\mathrm{X}$ : beide gucken auf den bereits von Ka geschriebenen Text; $M$ zeigt mit ihrem Stift drauf; ab Kas Sprechen gucken sich...

M: d'abord il faut se présenter un peu,. dans le premier paragraphe'... après äh. écrire quelque chose sur DANONE äh

$\mathrm{Ka}$ : äh je suis (Orientierung zu M)

X: beide an, ab Ms Einwand wieder Orientierung auf den Text, auf dem Stift einzelne Bereiche markiert werden

M: après. äh... oui mmh oui VOILA

$\mathrm{Ka}$ : demander tout simplement, d'abord on peut écrire que tu es étudiante (3) que $\mathbf{t}^{\prime}$ as vu l'annonce 
$\mathrm{X}$ : gucken sich an, M läßt den Stift fallen; Ka markiert jetzt mit ihren Fingern auf dem Text Stellen

M: voilà oui

Ka: ici, à l'université,... et que tu es très intéressée et ((leise)) connais et quelquechose,...

$\mathrm{X}$ : Geste von Ka für eine imaginäre Wand

Hier ist die bereits schon skizzierte „polnische“ Aufteilung zu beobachten. Magdalene bewirbt sich, Katharina hat dafür das Aufschreiben übernommen. Nachdem zunächst mit einer Formulierung begonnen wird, scheint es für Magdalena wichtig zu sein, die Globalstruktur zu referieren, wobei sie ein klares Paragraphenmuster vor Augen hat, dass ihr auch von Katharina bestätigt wird, die bereits an einzelne Elemente erinnert. Die klare und schnelle Bestätigung von Magdalene (voilà) zeigt, dass gemeinsames Wissen vorhanden ist, an das angeknüpft werden kann. Beide wissen, wie es geht und was von ihnen in dieser Situation verlangt wird. Das gemeinsame Wissen erstreckt sich also nicht nur auf Formulierungen, wie in den ersten beiden Beispielen gesehen, sondern auf die ganze Struktur des Briefes.

\section{Und das Glück des Autors...?}

Dadurch, dass die polnischen Studierenden gemeinsam über ein Muster verfügen, das sie in ihren Sprachpraxiskursen gelernt haben, wird ihnen die gemeinsame Arbeit und die Formulierungsarbeit erleichtert. Sie wissen über das Wissen der anderen und sie wissen auch, was entsprechend ihres Musters ,richtig“ ist. Während ihrer Interaktion orientieren sie sich an ihrem Musterwissen. Dies führt zu einer erheblichen Entlastung, kann aber auch bei fehlenden Voraussetzungen (wie der Angabe der Annonce) zu Irritationen führen.

Bei den deutschen Studierenden sind die Voraussetzungen anders. Sie verfügen über kein gemeinsames Muster für eine französische Bewerbung. Ihre Kenntnisse sind aus anderen Zusammenhängen gewonnen als die ihrer Universitätsausbildung. Die Voraussetzungen innerhalb der Dyade können also auch unterschiedlich sein, was zu einer Herausbildung einzelner Expertisen führen kann. Die Lösung des Problems fehlender gemeinsamer Voraussetzungen führt dazu, dass die Beteiligten die Aufgabe stärker personalisieren und einen eigenen $\mathrm{Be}$ werbungskontext herstellen. Dies führt aber gleichermaßen zu einer stärkeren Verteilung der Zuständigkeit und Verantwortlichkeit innerhalb der Interaktion und zu erheblich längerem zeitlichen und inhaltlichen Aufwand bei der Lösung der Aufgabe. Die deutschen Paare müssen sich viel stärker über ihr Wissen austauschen und müssen viel stärker erst eine gemeinsame Orientierung herstellen. 
In den gemeinsamen, an die Aufgabe anschließenden Besprechungen äuBerten sich die polnischen Studierenden einheitlich, dass ihnen die Aufgabe und vor allem das gemeinsame Lösen der Aufgabe Spaß gemacht hätte. Die deutschen Studierenden hingegen waren weder mit dem Resultat noch mit der Herstellung besonders zufrieden. Sie beklagten insbesondere das fehlende Wissen über sprachlich „korrekte“ Formen. Ihnen fehlte eine Kontrollinstanz. In Einzelfällen führt dies dazu, dass das Textmuster deutscher Bewerbungsschreiben diese Funktion übernimmt. Dadurch stellt sich das beklagte Problem der vorgeformten Ausdrücke aber weiterhin und kann auch nicht durch entsprechende Recherchen im zweisprachigen Lexikon gelöst werden.

Das gemeinsame Musterwissen - wie hier bei den polnischen Studierenden zu beobachten - kann dazu führen, dass die Bearbeitung der Aufgabe deutlich gleichzeitig und zum Teil sogar synchron erfolgt, indem beispielsweise beide die Schreibaufgabe lesen, beide gleichzeitig Formulierungen wiederholen. Dies führt nicht nur zu einer Entlastung, sondern auch zu mehr Spaß bei der Arbeit. Die Gefahr liegt meiner Ansicht nach darin begründet, dass das Muster selbst keiner Überprüfung mehr ausgesetzt wird, und nicht nur zur Kontrolle, sondern auch zur Einhaltung zwingt und damit zur Aufgabe selbst wird. Das kann eine individuellere oder besser passende Lösung verhindern. ${ }^{15}$ Dies gelingt den deutschen Studierenden zwar besser, ihre Texte entsprechen aber nicht dem Typ französisches Bewerbungsschreiben, sie wären in einer realistischen Situation also möglicherweise wenig erfolgreich. Außerdem führt die mangelnde Kenntnis über ein entsprechendes Muster zu einer starken Belastung und Frustration der Dyaden. In der Fremdsprachendidaktik ist die Vermittlung von Textmustern daher durchaus sinnvoll, allerdings müsste verstärkt darauf hingewiesen werden, dass auch Textmuster variabel sind und variiert werden müssen.

\section{LITERATUR}

Adamzik, Kirsten; Antos, Gerd; Jakobs, Eva-Maria (1997): Domänen- und kulturspezifisches Schreiben. Frankfurt am Main: Peter Lang Verlag.

Brown, Penelope; Levinson, Stephen C. (1987): Politeness: some universals in language usage. Cambridge: Cambridge University Press.

Bouchard, Robert; Galumyn, Marie-Madeleine de (1997): Médiation verbale et processus rédactionnel: parler pour écrire ensemble. In: Grossen, Michèle; Py, Bemard (éd.): Pratiques sociales et médiations symboliques. Frankfurt am Main: Peter Lang Verlag, 153-173.

Büchle, Karin (1992): Produzieren von Brieftexten in der Fremdsprache Deutsch. In: Bōmer, Wolfgang; Vogel, Klaus (Hg.): Schreiben in der Fremdsprache: Prozess und Text, Lehren und Lernen. Bochum: AKS-Verlag, 93-108.

Clyne, Michael (1987): Cultural differences in the organization of academic texts. In: Journal of Pragmatics 11, 217-247.

\footnotetext{
${ }^{15}$ So charakterisieren sich die polnischen Studierenden in allen zehn Briefen als entreprenante.
} 
Clyne, Michael (1993): Pragmatik, Textstruktur und kulturelle Werte. Eine interkulturelle Perspektive. In: Schröder, Hartmut (Hg.): Fachtextpragmatik Tübingen: Gunter Narr Verlag, 3-18.

Connor, Ulla (1992): Contrastive Rhetoric. Cross-cultural aspects of second-language writing. Cambridge: Cambridge University Press.

Dausendschőn-Gay, Ulrich; Gülich, Elisabeth; Kraff, Ulrich (1992): Gemeinsam schreiben. Konversationelle Schreibinteraktionen zwischen deutschen und französischen Gesprächspartnern. In: Krings, Hans P.; Antos, Gerd (Hg.): Textproduktion. Neue Wege der Forschung. Trier: Wissenschaftlicher Verlag, 219-256.

Dausendschön-Gay, Ulrich; Krafft, Ulrich (1996): Prozesse interaktiven Formulierens: Konversationelles Schreiben in der Fremdsprache. In: Börner, Wolfgang; Vogel, Klaus (Hg.): Texte im Fremdsprachenerwerb. Verstehen und Produzieren. Tübingen: Gunter Narr Verlag, 253-274.

Dausendschön-Gay, Ulrich; Kraff, Ulrich (1999): Système écrivant et processus de mise en mots dans les rédactions conversationelles. In: Langages 34(134), 51-67.

Drescher, Martina (1994): Für zukünftige Bewerbungen wünschen wir Ihnen mehr Erfolg. Zur Formelhaftigkeit von Absagebriefen. In: Deutsche Sprache 22(2), 117-138.

Gajewska, Elzbieta (1998): Les méthodes de FLE pour adultes débutants face aux besoins de la communication professionelle. Praca doktorska, Poznań.

Gülich, Elisabeth (1997): Routineformeln und Formulierungsroutinen. Ein Beitrag zur Beschreibung formelhafter Texte. In: Wimmer, Rainer; Berens, Franz-Josef (Hg.): Wortbildung und Phraseologie. Tübingen: Gunter Narr Verlag, 131-175.

Gulich, Elisabeth; Krafft, Ulrich (1998): Zur Rolle des Vorgeformten in Textproduktionsprozessen. In: Wirrer, Jan (Hg.): Phraseologismen in Text und Kontext. Bielefeld: Aisthesis Verlag, 11-38.

Hempelmann, Claudia (1997): Bewerbungsbriefe als Balanceakt zwischen Formelhaftigkeit, Kreativität und Ratgeberliteratur. Ein deutsch-französischer Vergleich. Staatsexamensarbeit Bielefeld.

Kaplan, Robert (1966): Cultural Thought Patterns in Inter-Cultural Education. In: Language Learning 16, 1-20.

Kienzler, Iwona (1999): Korespondencja handlowa w jezyku polskim. Wzory pism, umów i innych dokumentów. Gdansk: ATEXT S.A.

Krings, (1986): Wie Lemer Texte machen. Schreibprozesse im Licht introspektiver Daten. In: Probleme und Perspektiven der Sprachlehrforschung. Bochumer Beiträge zum Fremdsprachenunterricht. In: Forschung und Lehre. Frankfurt am Main: Skriptor, 257-280.

Krings, Hans P. (1989): Schreiben in der Fremdsprache - Prozeßanalysen zum ,vierten skill‘. In: Antos, Gerd; Krings, Hans P.: Textproduktion: Ein interdisziplinärer Forschungsüberblick. Tubingen: Niemeyer, 377-431.

Krings, Hans P. (1992): Empirische Untersuchungen zu fremdsprachlichen Schreibprozessen - Ein Forschungsüberblick. In: Bömer, Wolfgang, Vogel, Klaus (Hg.): Schreiben in der Fremdsprache: Prozess und Text, Lehren und Lemen. Bochum: AKS Verlag, 47-77.

Lehnen, Katrin (1999): Kooperative Textproduktion. In: Kruse, Otto; Jakobs, Eva-Maria; Ruhmann, Gabriele (Hrsg.): Schlüsselkompetenz Schreiben. Konzepte, Methoden, Projekte für Schreibberatung und Schreibdidaktik an der Hochschule. Neuwied u.a.: Luchterhand, 147-169.

Lehnen, Katrin (2000): Kooperative Textproduktion. Zur gemeinsamen Herstellung wissenschaflicher Texte im Vergleich von ungeübten, fortgeschrittenen und sehr geübten SchreiberInnen. Dissertation, Bielefeld.

Limbrunner, Christiane (1999): Schriftliche Bewerbungen in Europa - eine kontrastive Studie mit einem Bewerbungsleitfaden für Deutschland. Magisterarbeit, Bielefeld.

Pogner, Karl-Heinz (1999): Schreiben im Beruf als Handeln im Fach. Tübingen: Gunter Narr.

Purves, Alan C. (ed.) (1988): Writing across languages and cultures. Issues in Contrastive Rhetoric. Newbury Park: SAGE Publications. 
Sandig, Barbara (1997): Formulieren und Textmuster. Am Beispiel von Wissenschaftstexten. In: Jakobs, Eva-Maria; Knorr, Dagmar (Hrsg.): Schreiben in den Wissenschaften. Frankfurt am Main: Peter Lang Verlag, 25-44.

Tiittula, Liisa (1995): Kulturelie Unterschiede im mündlichen und schriftlichen Gebrauch von Sprache. In: Raible, Wolfgang (Hg.): Kulturelle Perspektiven auf Schrift und Schreibprozesse. Elf Aufsătze zum Thema Mündlichkeit und Schriftlichkeit. Tübingen: Gunter Narr Verlag, 233-257.

\section{TRANSKRIPTIONSKONVENTIONEN}

..... Pause, die Anzahl der Punkte entspricht der relativen Dauer.

, z.B. genau, $\quad$ Pause, Zahl in Klande Melodie

hallo* steigende Melodie

GROB auffällige Betonung eines Wortes, einer Silbe, eines Lautes z.B. HAllo

((schnell)) Charakterisierung der Sprechweise

$+\quad$ Ende des Gültigkeitsbereiches einer Charakterisierung

$X \quad$ besondere Zeile für die Kommentierung von nichtsprachlichen Ereignissen

Le Groupe Danone est un des leaders de l'industrie alimentaire mondiale. Fondé en 1966, les produits du Groupe Danone - produits laitiers frais, biscuits, boissons mais aussi plats cuisinés surgelés - sont présents dans tous les pays du monde.

$\rightarrow$ Savez-vous que le concept DANONE fut crée dans la région lyonnaise avec la fusion de deux entreprises verrières, Boussois et Souchon-Neuvesel (BSN)?

$\rightarrow$ Savez-vous que le BSN et Gervais Danone ont annoncé leur fusion en 1973 et sont devenus d'un seul coup le premier groupe alimentaire français? ...

$\rightarrow$ Savez-vous que DANONE est aujourd'hui une de quinze sociétés françaises cotées à la Bourse de New York et le numéro un mondial des produits laitiers frais?

Si vous voulez en savoir plus sur cette entreprise internationale, sur sa diversité, son enthousiasme et son respect des hommes et de l'environnent, vous avez maintenant l'occasion! DANONE vous offre un stage de deux à six mois dans ses sièges à Lyon ou à Paris.

Vous êtes un(e) jeune étudiant(e) européen(e) parlant couramment le français!

Vous êtes entreprenant, ouvert et curieux de connaître cette entreprise extraordinaire!

Envoyez-nous une lettre de motivation où vous nous direz ce que vous attendez de votre stage et où vous expliquerez pourquoi vous êtes le parfait complément pour notre team jeune et motivé.

Envoyez votre candidature à:

Recrutement des jeunes étudiants

Groupe DANONE

7 , rue de Tehéran

F-75381 Paris Cedex 08 